\title{
Magnetic energy and magnetic helicity budget in AR 8210: What are the sources of flaring activity?
}

\author{
S. Régnier ${ }^{1}$, R. C. Canfield ${ }^{2}$ \\ ${ }^{1}$ ESTEC/ESA, Keplerlaan 1, 2201 AZ Noordwijk, The Netherlands email: sregnier@rssd.esa.int \\ ${ }^{2}$ Physics Department, 264 EPS Building, Montana State University, Bozeman, MT 59715 USA \\ email: canfield@solar.physics.montana,edu
}

\begin{abstract}
The active region 8210 (AR 8210) observed on May 1, 1998 is the site of numerous flares. By studying a time series (from 17:00 UT to 21:40 UT) of reconstructed coronal magnetic configurations, we give a scenario of the eruptive phenomena in AR 8210 involving the rotation of the sunspot and the complex topology in a reconnection process. We also study the time evolution of the energy and of the helicity budget. We compute the free energy in the corona, the magnetic energy rate due to transverse photospheric motions. We conclude that the photospheric motions are related to flaring activities and occur before the injections of energy into the corona. In terms of magnetic helicity, we are able to derived the relative magnetic helicity (relative to the potential field) and the helicity of the potential field configuration. The vacuum helicity (or helicity of the potential field) is constant during the time period and characterizes the complex topology. For this time period, the relative magnetic helicity does not show obvious changes related to the flaring activity.
\end{abstract}

\section{Introduction}

To understand the eruptive phenomena in solar corona, we need to know the 3D magnetic configuration of active regions. Currently one reliable way to do so is to extrapolate the magnetic field into the corona from photospheric measurements. In previous studies (Régnier et al. 2002, Régnier \& Amari 2004), we have shown that the coronal magnetic configuration of active regions can be well determined by using a nonlinear force-free (nlff) field extrapolation (Amari et al. 1997). We here extend the method to the time evolution of an active region exhibiting an important flaring activity. The questions we would like to answer are: is the magnetic topology stable inside the reconstructed magnetic configurations during the time period? Are the evolutions of the magnetic energy and of the magnetic helicity consistent? What are the main changes before and after a flare?

\section{Magnetic field configurations}

We study AR 8210 observed on May 1, 1998 between 17:00 UT and 21:40 UT. During the time period, 5 C-class flares were observed by both the GOES- 8 satellite measuring the integrated solar X-ray flux and EIT/SOHO which allows us to determine the sources of the flares on the disk. To reconstruct the magnetic field into the corona, we use a time series of 15 photospheric vector magnetograms obtained by IVM (Imaging Vector Magnetograph). The long-term evolution of AR 8210 shows that the sunspot is rotating clockwise. We focus our study on the South-East location where the sunspot rotation increases the shear and where most of the flares originates (see Régnier \& Canfield 
2004) The magnetic configuration around the high sheared region looks like a three source configuration as described by Brown \& Priest (1999): one negative polarity (the sunspot) and two positive polarities. A separatrix surface divides this configuration into two domains of connectivity (each domain containing one positive polarity and a part of the negative one). The magnetic flux of this system is almost balanced. When the sunspot is rotating clockwise, the field lines from one connectivity domain are closer and closer to the separatrix surface and then a reconnection process takes place which reconnects the field lines in the other connectivity domain. The time of the reconnection can be estimated using the blueshift events occuring near the high shear region observed in the $\mathrm{H} \alpha$ line by the MCCD (MSO). The blueshift events and the flares and therefore the reconnection process are closely related.

\section{Magnetic energy and magnetic helicity}

From the photospheric vector magnetograms, we compute the magnetic energy rate due to transverse motions (see e.g. Kusano et al. 2002). From the 3D magnetic configuration, we derive the magnetic energy inside the coronal volume. We conclude that the injection of magnetic energy through the photosphere occur before (or at the same time) as the flare and the coronal changes of magnetic energy due to the flares.

The vector potential Grad-Rubin-like method allows us to have access to the vector potential, $\vec{A}$ in the volume. Therefore we can easily derive different magnetic helicity: the relative magnetic helicity given by the Berger \& Field formula (1984) and the vacuum helicity which is the helicity of the potential field (see Berger 1999). The relative magnetic helicity is in averaged negative during the time period. The comparison with the GOES $\mathrm{X}$-ray flux shows that before the flares negative helicity is injected into the corona. For the time series, we obtain that the vacuum helicity is constant. The vacuum helicity characterizes the basic topology of the magnetic configurations. As the vacuum helicity is constant, we can conclude that the topology of the $3 \mathrm{D}$ coronal field remains almost unchanged during its evolution.

\section{Conclusions}

We conclude that we can follow the time evolution of the coronal field by successive nonlinear force-free equilibria and that the main phenomena which are precursors of flares are the photospheric motions close to topological elements (e.g. separatrix surfaces).

\section{Acknowledgements}

This work was supported by AFOSR, under a DoD Multi-Universities Research Initiative (MURI) grant. This work is now a part of the European Solar Magnetism Network (EC contract HPRN-CT-2002-00313).

\section{References}

Amari, T. et al. 1997, Solar Phys., 174, 129-149.

Berger, M. A., Field, G. B1984, Journal of Fluid Mechanics, 147, 133-148.

Berger, M. A.1999, in Magnetic Helicity in Space and Laboratory Plasmas Eds Brown et al. Brown, D. S., Priest, E. R. 1999, Solar Phys., 190, 25.

Kusano, K., Maeshiro, T., Yokoyama, T., Sakurai, T. 2002, ApJ, 577, 501.

Régnier, S., Amari, T., Kersalé, E. 2002, A\&A A, 392, 1119-1127.

Régnier, S., Amari, T. 2004, $A \mathscr{E} A$, in press.

Régnier, S., Canfield, R. C. 2004, in preparation. 